\title{
Self-assembly of organic molecules at metal surfaces
}

\author{
Angelika Kühnle* \\ Fachbereich Physik, Universität Osnabrück, Barbarastr. 7, 49076 Osnabrück, Germany \\ Received 8 October 2007; received in revised form 2 January 2008; accepted 3 January 2008 \\ Available online 15 January 2008
}

\begin{abstract}
Self-assembly represents a promising strategy for surface functionalisation as well as creating nanostructures with well-controlled, tailor-made properties and functionality. Molecular self-assembly at solid surfaces is governed by the subtle interplay between molecule-molecule and molecule-substrate interactions that can be tuned by varying molecular building blocks, surface chemistry and structure as well as substrate temperature.

In this review, basic principles behind molecular self-assembly of organic molecules on metal surfaces will be discussed. Controlling these formation principles allows for creating a wide variety of different molecular surface structures ranging from well-defined clusters, quasi onedimensional rows to ordered, two-dimensional overlayers. An impressive number of studies exist, demonstrating the ability of molecular selfassembly to create these different structural motifs in a predictable manner by tuning the molecular building blocks as well as the metallic substrate.

Here, the multitude of different surface structures of the natural amino acid cysteine on two different gold surfaces observed with scanning tunnelling microscopy will be reviewed. Cysteine on $\mathrm{Au}(110)-(1 \times 2)$ represents a model system illustrating the formation of all the above mentioned structural motifs without changing the molecular building blocks or the substrate surface. The only parameters in this system are substrate temperature and molecular coverage, controlling both the molecular adsorption state (physisorption versus chemisorption) and molecular surface mobility. By tuning the adsorption state and the molecular mobility, distinctly different molecular structures are formed, exemplifying the variety of structural motifs that can be achieved by molecular self-assembly.
\end{abstract}

(C) 2008 Elsevier Ltd. All rights reserved.

Keywords: Self-assembly; Organic molecules; Cysteine; Thiol; Metal surfaces; Scanning tunneling miscroscopy

\section{Introduction}

Modern material science is based upon providing smart solutions to a wide range of different applications in very heterogeneous environments. Ideally, functional materials should be tailored to address specific tasks and to provide well-defined functionality such as molecular recognition for (bio)sensors or protective layers for surface coatings [1].

Molecular self-assembly has been recognised as a very promising strategy for creating such tailor-made functional materials. Especially at solid surfaces, extended, two-dimensional molecular layers are already widely used, providing well-controlled properties such as corrosion resistance, surface superhydrophobicity, or antifouling coatings [2-4].

\footnotetext{
* Tel.: +49 541969 2642; fax: +49 54196912642.

E-mail address: kuehnle@uos.de.
}

However, molecular self-assembly bears the potential for engineering much more advanced structures with high degree of complexity. Recently it has been demonstrated that molecular self-assembly can be employed to fabricate surface structures ranging from clusters $\left[5^{\bullet}\right]$, unidirectional rows $\left[5^{\bullet}, 6^{\bullet}, 7\right]$ to porous networks with well-defined pore size and periodicity $\left[8^{\bullet \bullet}, 9^{\bullet}\right]$ or arrays of metal atoms $\left[10^{\bullet \bullet}\right]$. This exceptional flexibility opens up another emerging field of application, namely molecular electronics that is believed to be the successor of today's electronics based on silicon technology: As early as 1965 Moore has stated his famous "Moore's law" [11], predicting that the growing demand for cheap, small, but yet faster computers and other electronic devices will inevitably result in the end of the "silicon road" [12] as the needed miniaturisation of integrated circuits is limited to several tens of nanometres using conventional silicon technology based on socalled "top-down" lithography [13]. A promising solution to 
this challenge is integrating functional molecules into silicon devices as building blocks for e.g. wires, switches and transistors using "bottom-up" techniques. Nowadays, advances in chemistry synthesis allow to tailor the atomic structure of organic molecules providing a very wide range of flexibility in electronic structure. However, there is still a considerable gap between synthesising individual molecules with tailored electronic structure and an electronic circuit that can be addressed and connected to other devices. Besides the question of creating an interface to the macroscopic world, one of the main challenges for molecular electronics is to precisely position the molecular building blocks such that they eventually form a functioning structure like wires and logic operators. Manipulation of individual molecules one-by-one seems at present far too time-consuming to constitute a rational pathway for mass production of molecular electronic devices. Therefore, a technique that provides a route for creating molecular cluster, one-dimensional wires and even more complex structures in a predictable and well-controlled manner is of outmost importance for the evolution of molecular electronics. In contrast to addressing each molecule individually, employing molecular self-assembly has been identified as one of the few promising strategies for the mass fabrication of complex molecular systems $\left[14,15^{\bullet}, 16\right]$.

In order to take advantage of this versatile technique, a precise understanding of the mechanisms behind self-assembly is needed to enable the fabrication of well-defined molecular structures in a predictable manner. Consequently, molecular self-assembly has been studied extensively in the last decade. Many of these studies have been carried out on single crystal metallic or semiconducting surfaces with scanning tunnelling microscopy (STM) under ultra-high vacuum (UHV) conditions, as several central structuring motifs are experimentally accessible only when studied under clean and very precisely controlled UHV conditions. However, a growing number of publications emerge demonstrating a high degree of control in molecular self-assembly at the liquid-solid interface as well, especially on highly oriented pyrolytic graphite (HOPG) $\left[17^{\bullet}, 18\right]$.

In this review, the basic principles explaining molecular selfassembly on metal surfaces under UHV conditions will be summarised. These principles will be illustrated using cysteine on gold as a model system, demonstrating how distinctly different structures ranging from clusters and dimers, quasi onedimensional rows to two-dimensional overlayers can be achieved by changing the substrate temperature and the molecular coverage.

\section{Basic principles}

Molecular self-assembly has been defined as the "spontaneous assembly of molecules into structured, stable, noncovalently joined aggregates under equilibrium" $\left[19^{\circ}\right]$. This definition comprises the existence of predefined building blocks that interact weakly in order to provide the possibility for both bond formation and bond breaking until the stable equilibrium structure is formed. In contrast, in many experimental studies the system may not be able to reach the equilibrium state but might be trapped in a kinetically limited state. The term selforganisation has been established for such kinetically limited systems $\left[15^{\bullet}\right]$. The difference between molecular self-assembly and self-organisation is illustrated in Fig. 1: To distinguish between self-organisation and self-assembly the ratio between molecular flux and surface diffusivity needs to be considered. If the flux is high and diffusivity is low, the molecules are not able to reach their equilibrium structure, but rather get trapped in a diffusion-limited state, as demonstrated by the growth of dendrimeric Ag islands on $\mathrm{Pt}(111)$ [20]. On the other hand, if the flux of molecules is low and the diffusivity is high, the molecules are able to move freely on the surface, resulting in the growth of the thermodynamically favoured, equilibrium structure.

The structure formation is governed by the balance between intermolecular and molecule-substrate interactions as illustrated in Fig. 2a. The diffusivity of the molecules on the surface can be controlled by a variation of substrate temperature. Upon increasing the substrate temperature, thermal energy is transferred to the molecules, thereby providing sufficient kinetic energy $E_{\text {kin }}$ to overcome the diffusion barrier $E_{\mathrm{d}}$ on the surface (see Fig. 2b). This is one prerequisite for the formation of the thermodynamic equilibrium structure. On the other hand, the kinetic energy of the molecules $E_{\text {kin }}$ must, of course, not exceed the binding energy $E_{\mathrm{b}}$ of the molecules on the surface otherwise the molecules would desorb from the surface. Finally, we need to consider the intermolecular interaction energy $E_{\text {inter }}$. This interaction is crucial for the formation of ordered structures as it comprises the information on how to assemble the predefined building blocks. As mentioned above, the intermolecular interaction should be "weak", meaning that the molecular entities must possess sufficient energy to probe the corrugation of the energy landscape in order to identify the equilibrium structure in the global minimum. If the intermolecular interaction is "strong" the molecules stick together irreversibly once they have met, preventing the formation of an ordered equilibrium structure. On the other hand, the interaction between the molecules must be of sufficient strength to allow for the formation of a stable structure. The latter condition can be achieved when the intermolecular interaction energy $E_{\text {inter }}$ is of the same order as the kinetic energy of the molecules $E_{\text {kin }}$, being only slightly larger than $E_{\text {kin. }}$. The energy conditions to be fulfilled for molecular self-assembly can therefore be summarized as $E_{\mathrm{b}}>E_{\text {inter }} \geq E_{\text {kin }}>E_{\mathrm{d}}$.

An additional aspect needs to be considered when the molecules can both physisorb and chemisorb on the surface as depicted in Fig. 3. Usually diffusion barriers become very large when molecules chemisorb onto the surface as chemisorption is stronger than physisorption (Fig. 3a). An ordered structure of chemisorbed molecules on a substrate surface is, therefore, usually not governed by intermolecular interactions but solely by the strong chemisorption energy. This is why covalently bound molecules are usually not considered as molecular selfassembled structure, but rather as a "classical" superstructure (typical examples are atoms or small molecules like oxygen or carbon monoxide on various metal surfaces forming regular 


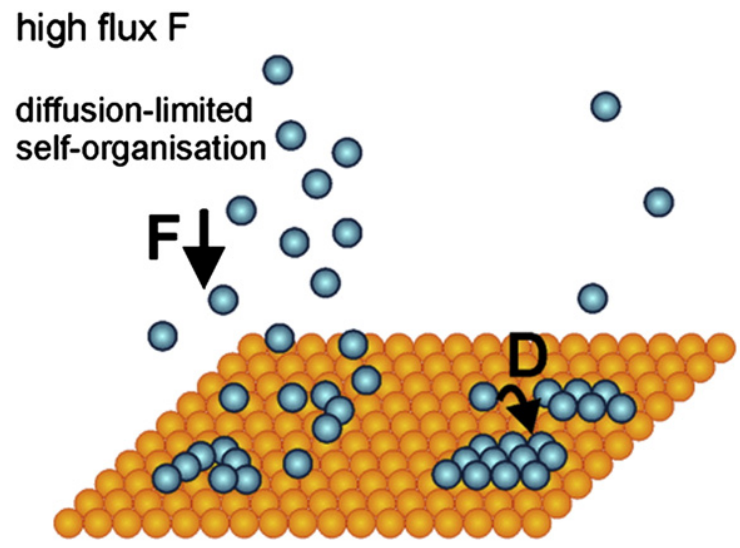

high diffusivity $D$

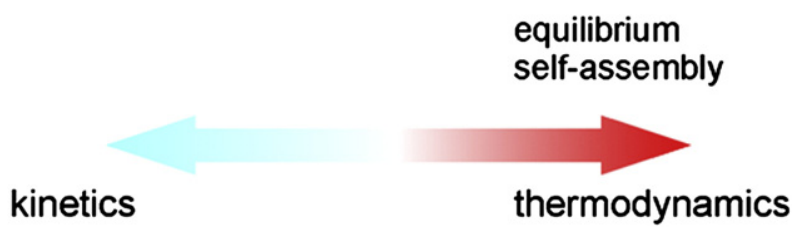

Fig. 1. Schematic illustrating the difference between self-organisation and selfassembly (adapted from $\left[15^{\circ}\right]$ ). Depending on the flux of incoming molecules and the diffusivity of the molecules on the surface, either diffusion-limited selforganised structures or self-assembled structures are formed. Self-organised structures form when the flux is high and diffusivity is low. These structures are governed by the kinetics of the system and can be far from equilibrium (to the left). On the other hand, when the flux is low and diffusivity is high, selfassembled structures at the thermodynamic equilibrium are formed (to the right).

superstructures). However, molecular self-assembly of chemisorbed species is possible in special cases, for example when the mobility of the substrate atoms is high enough such that the chemisorbed molecule together with the bound substrate atom forms a new entity, which then can diffuse and act as a new building block [21]. This is a rather common effect upon molecular adsorption onto metal surfaces: molecule deposition has been observed to cause both local substrate restructuring [22] as well as large-scale surface refacetting [23]. Interestingly, the archetypical example of self-assembled monolayers (SAMs), namely alkanethiols on $\mathrm{Au}(111)$ [24 ${ }^{\bullet}$, involve a covalent sulphur-gold bond. However, several indications exist $[21,25]$ that suggest a restructuring of the underlying gold substrate in line with the above picture of a diffusing moleculesubstrate atom entity.

As shown in Fig. 3b, an activation barrier $\Delta E$ can exist between the weakly bound physisorbed molecule and the chemisorbed state. If thermal energy is not sufficient to overcome the barrier between the physisorbed and the chemisorbed state, the molecules remain physisorbed on the surface, allowing for investigating the self-assembled structure of the physisorbed species. Upon increasing the substrate temperature, the energy barrier might be overcome, resulting in chemisorbed molecules on the surface. These molecules can, as outlined above, again form self-assembled structures, which can deviate significantly from the physisorbed species due to the substantial change in the molecular building block.
Another example that requires activation energy is dehydrogenation necessary to allow hydrogen bond formation. In a recent study, 4,9-di-aminoperylene-quinone-3,10-di-imide (DPDI) was annealed to $570 \mathrm{~K}$ in order to remove hydrogen from the molecule $\left[26^{\bullet \bullet}\right]$. The remaining residuals are now activated in a sense that the intermolecular interaction (hydrogen bonding) is "switched on" after the dehydrogenisation.

Besides the energetics discussed above, also the molecular building blocks can be tailored for creating well-defined molecular structures. Organic molecules as building blocks provide a wide range of variety regarding both strength of interaction as well as directionality. Moreover, they can be synthesised with great flexibility and control. This is why organic molecules have become the standard for molecular selfassembly experiments. Of special interest are biomolecules such as amino acids and DNA bases, the latter are very attractive for

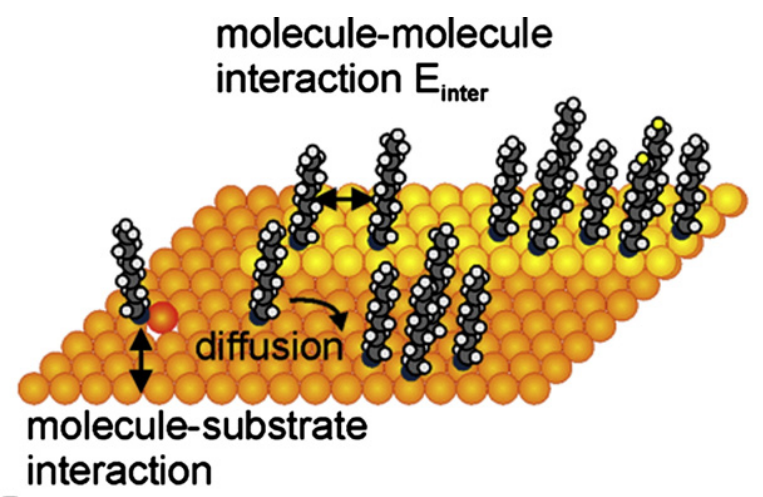

(a)

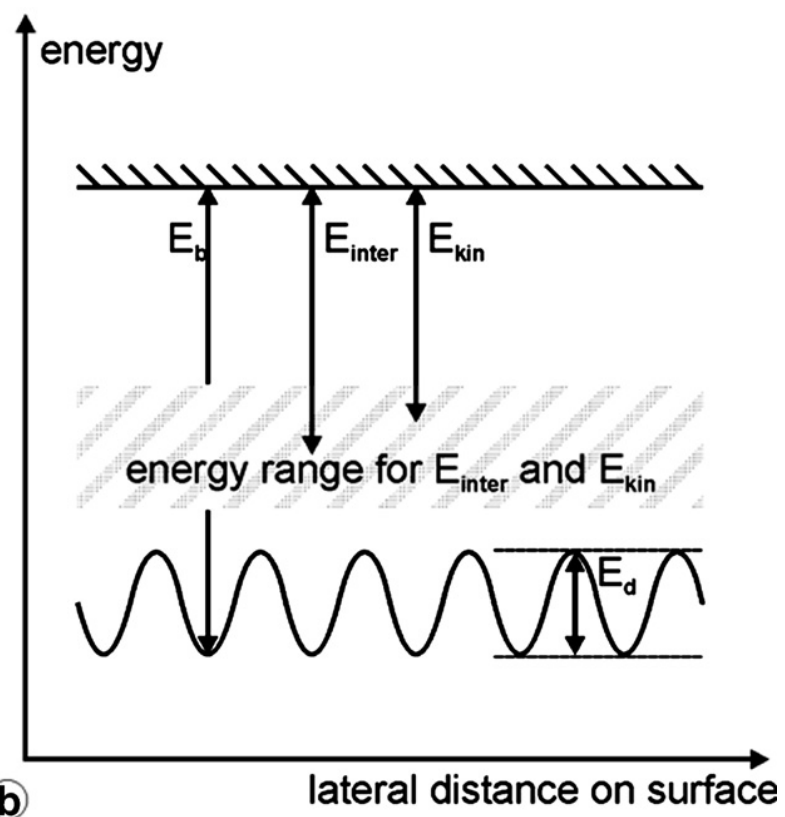

Fig. 2. Mechanisms controlling molecular self-assembly on surfaces. a) The subtle interplay between molecule-molecule and molecule-surface interactions governs the structure formation and can be employed for creating tailor-made structures. b) For molecular self-assembly to occur, the energies involved $\left(E_{\mathrm{b}}\right.$ binding energy of the molecules to the surface; $E_{\mathrm{d}}$ diffusion barrier of the molecules on the surface, $E_{\text {inter }}$ intermolecular interaction energy, $E_{\text {kin }}$ kinetic energy of the molecules) must obey the energy condition $E_{\mathrm{b}}>E_{\mathrm{inter}} \geq E_{\mathrm{kin}}>E_{\mathrm{d}}$. 

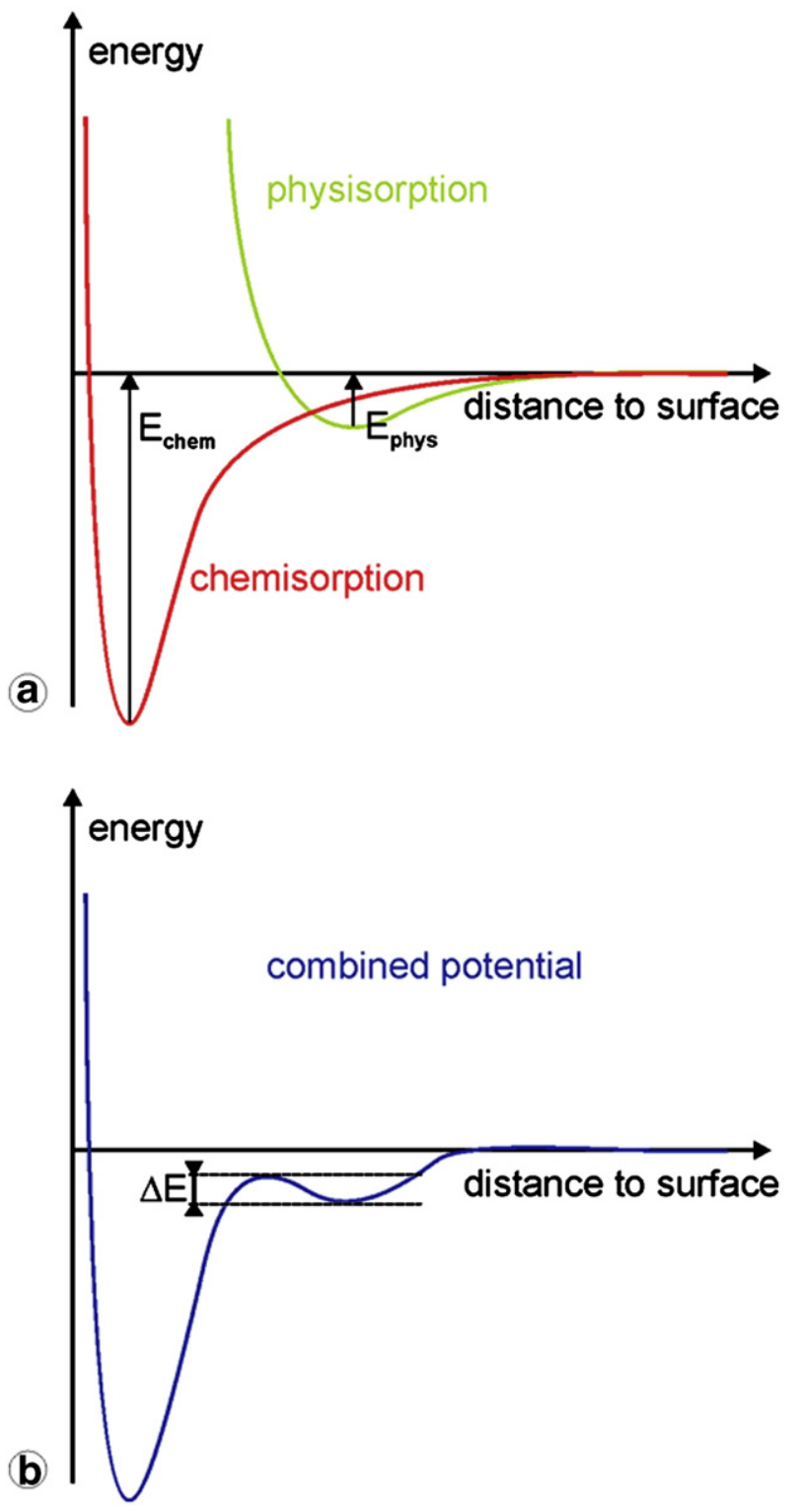

Fig. 3. Schematic potential energy diagram illustrating the difference between physisorbed and chemisorbed species. a) Physisorption takes place at larger distance $d>0.3 \mathrm{~nm}$ and is associated with weak van der Waals interaction energies around $0.1 \mathrm{eV}$. Chemisorption, in contrast, originates from the formation of strong covalent or ionic bonds at shorter distances. b) In the combined potential, a barrier might exist that needs to be overcome for chemisorption to occur.

molecular self-assembly due to their ability to selectively form hydrogen bonds with well-controlled direction, which is defined by the molecular structure $\left[27^{\bullet}\right]$.

The basic molecule-molecule interaction types to be considered for molecular self-assembly are listed in Table 1. It is instructive to classify the different interaction types in terms of interaction strength, typical bonding length and nature $\left[16^{\bullet \bullet}\right]$. As already mentioned, hydrogen bonds are an interesting interaction for self-assembly as they provide both molecular selectivity and well-defined orientation. Also dipoledipole interaction can lead to very selective and thus wellcontrolled structure formation. A prime example demonstrating the potential of dipole-dipole interaction for molecular selfassembly has been reported by Yokoyama et al. [28 ${ }^{\bullet \bullet}$. By varying the number and position of substituents on a porphyrin molecule the structure formation can be controlled in a predictable fashion. Besides these two bonding types, also metal atom complexation can provide a selective and directed interaction [29]. The main other interactions, namely van-derWaals and electrostatic interactions, are non-selective. Although van-der-Waals interactions are comparatively weak, they can have a significant influence on molecular self-assembly of organic molecules possessing e.g. long alkane chains.

In addition to these direct intermolecular interactions, it is also possible that molecules communicate indirectly through substrate-mediated interactions. An example for substratemediated interaction is the oscillatory interaction induced by the two-dimensional electron gas of the surface state, having an interaction length of up to $7 \mathrm{~nm}\left[6^{\bullet}, 30^{\bullet \bullet}\right]$. Substrate-mediated repulsion is also the key for understanding the network formation of anthraquinone molecules on $\mathrm{Cu}(111)\left[9^{\bullet}\right]$. However, the detailed nature of this repulsion is not completely understood at present.

Another substrate-mediated interaction mechanism can be caused by adsorbate-induced reconstructions. Molecular structure formation can be directed e.g. to form unidirectional rows although the individual entities do not interact along the row direction [31]. This can be understood by the fact that the energy for extending an existing reconstruction is typically less than forming an entirely new reconstruction structure.

Another important aspect to be considered is the competition of different intermolecular interaction types such as isotropic van-der-Waals interactions and anisotropic hydrogen bondings [32]. Recent theoretical investigations revealed that a kinetically-controlled transition exists between the growth of compact islands and one-dimensional molecular chains, resulting in a distinct temperature regime for one-dimensional growth [32].

Finally, also the molecular coverage influences the final structure to be formed. A nice example is self-assembly of dehydrogenated DPDI on $\mathrm{Cu}(111)$, which reveals three distinctly different structures in dependence on the coverage $\left[26^{\bullet \bullet}\right]$. At low coverages, an open honeycomb network is formed that is only stable up to a maximum coverage of 0.7 monolayer $(\mathrm{ML})$. In an intermediate coverage regime of

Table 1

Intermolecular interaction types with typical interaction energies and bonding lengths

\begin{tabular}{llll}
\hline Interaction type & Strength & Bonding length & Nature \\
\hline van-der-Waals & $\sim 0.1 \mathrm{eV}$ & $0.5 \ldots 1 \mathrm{~nm}$ & Non-selective \\
H bonding & $0.1 \ldots 0.5 \mathrm{eV}$ & $0.20 \ldots 0.35 \mathrm{~nm}$ & Selective, directed \\
Electrostatic & $0.1 \ldots 3 \mathrm{eV}$ & Up to several nm & Non-selective \\
Dipole-dipole & $0.1 \ldots 0.5 \mathrm{eV}$ & $0.2 \ldots 0.3 \mathrm{~nm}$ & Directed \\
Metal complexation & $1 \ldots 3 \mathrm{eV}$ & $0.2 \ldots 0.3 \mathrm{~nm}$ & Selective \\
Substrate-mediated & $0.1 \ldots 1 \mathrm{eV}$ & up to $7 \mathrm{~nm}$ & Oscillatory \\
$\begin{array}{l}\text { Reconstruction- } \\
\quad\end{array}$ & $1 \mathrm{eV}$ & system dependent & Covalent \\
\hline
\end{tabular}

Depending on the interaction type, bonding energy, distance and nature can be very different. These differences can be exploited for controlling molecular selfassembly. 
$0.85 \mathrm{ML}$, an extended layer assembled by a trimeric unit is formed. When a complete monolayer is deposited, the molecules arrange themselves in a dense packing which is incommensurate with the underlying substrate.

Recent developments include coadsorption or sequential

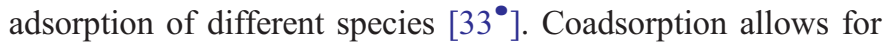
the formation of even more advanced structures such as a network formed by one type of molecule that represents the

\section{a extended, two-dimensional layer}

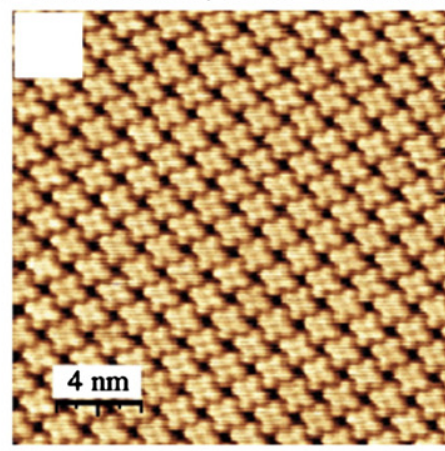

b quasi one-dimensional structure
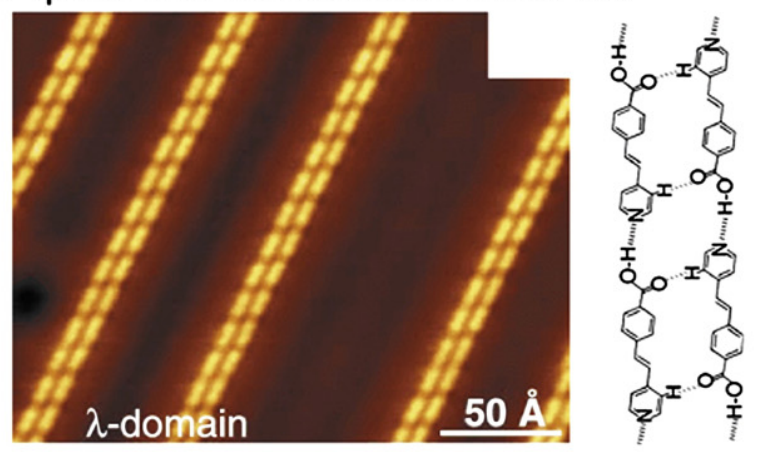

\section{c clusters}
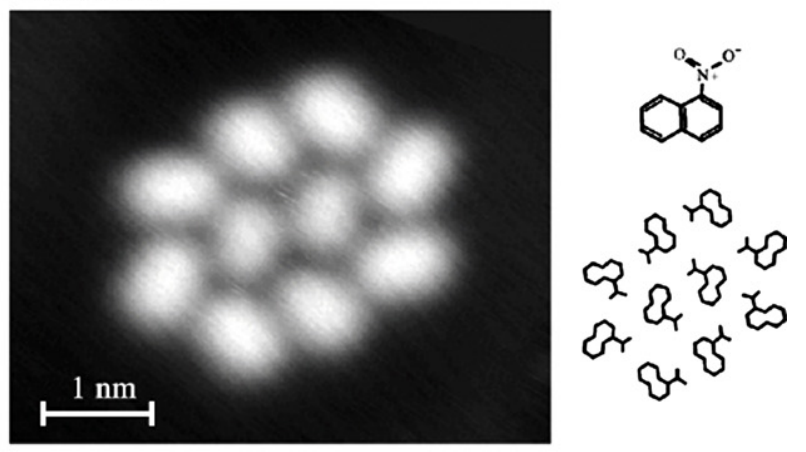

\section{d porous network}

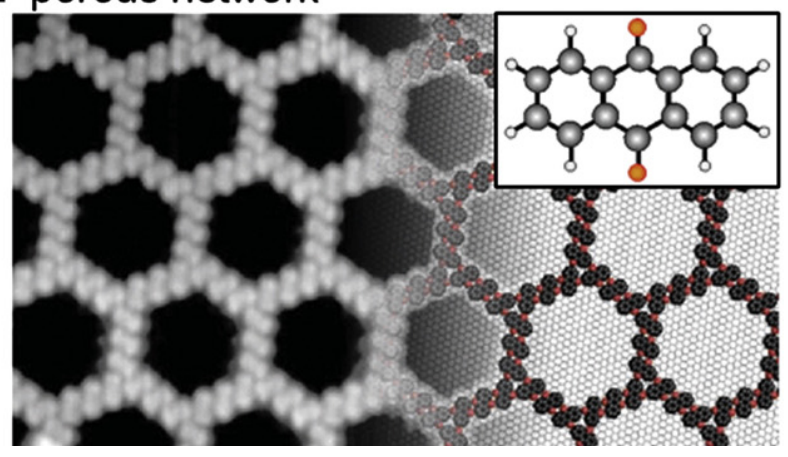

"host" for a "guest" molecule. A very elegant example for such a host-guest system is an H-bonded network based on perylene tetra-carboxylic di-imide (PTCDI) and melamine linkers with $\mathrm{C}_{60}$ molecules as guest molecules $\left[8^{\bullet \bullet}\right]$. The structure formation of such coadsorbed systems has been addressed theoretically by Monte Carlo simulations. Depending on the specific interaction energies and molecular coverage, these simulations reveal whether the two molecular species segregate or form mixed structures [34].

Employing and combining the above mentioned strategies in a clever way have led to the achievement of impressive control and predictability in molecular self-assembly. Recent advances have demonstrated a great flexibility in structure formation as illustrated in Fig. 4. A considerable number of structures observed upon molecular self-assembly at solid surfaces comprise extended, two-dimensional molecular monolayers (Fig. 4a) [35-39]. These systems typically have in common that the intermolecular interaction dominates over the moleculesubstrate interaction. Especially for large organic molecules it has been recognised that incommensurate or so-called "quasiepitaxial" film growth is more likely than epitaxial growth, which can be understood by the increasing number of energetically similar adsorption sites when increasing the size of the molecule. A prominent exception to this rule of thumb is perylene tetra-carboxylic di-anhydride (PTCDA) on $\operatorname{Ag}(111)$, which forms perfectly ordered commensurate overlayers $\left[40^{\circ}\right]$.

Besides extended layers, also quasi one-dimensional, molecular chains have been observed, using the control of intermolecular interactions like hydrogen bonding $\left[5^{\bullet}, 7,28^{\bullet \bullet}, 41^{\bullet \bullet}\right]$ (Fig. 4b) or substrate-mediated interactions $\left[6^{\bullet}, 31\right]$. Two other studies might be mentioned in this context although they deviate slightly from the scope of this review as they involve the activation of directed growth by a voltage pulse of the STM tip $[42,43]$. In these studies, adsorbed organic molecules undergo a polymerisation reaction after initial activation, forming very well-defined, one-dimensional molecular chains on the surface.

Other structures such as molecular clusters (Fig. 4c) like trimers, tetramers $\left[28^{\bullet \bullet}\right]$ and decamers $\left[5^{\bullet}\right]$ or porous networks $\left[8^{\bullet \bullet}, 9^{\bullet}\right]$ (Fig. 4d) as well as even more complex structures involving regular arrays of metal ions that are kept in place by molecular entities [44] have now been realised by the clever design of the molecular building blocks.

So far, the vast majority of self-assembly experiments involving organic molecules have been carried out on single

Fig. 4. Advances in molecular self-assembly. a) Extended, two-dimensional overlayer of octafluoro zinc phthalocyanine $\left(\mathrm{ZnPcF}_{8}\right)$ molecules on $\mathrm{Ag}(111)$. Image size: $20 \mathrm{~nm} \times 20 \mathrm{~nm}$. Reprinted figure with permission from [39]. Copyright 2007 by the American Physical Society. b) Quasi one-dimensional structures. Unidirectional molecular rows self-assembled of 4-[trans-2-(pyrid4-yl-vinyl)]benzoic acid (PVBA, see insert) on $\operatorname{Ag}(111)$. Reprinted figure with permission from [7]. Copyright 2001 by the American Physical Society. c) Self-assembled molecular clusters of 1-nitronaphtalene (NN) on $\mathrm{Au}(111)$. Reprinted figure with permission from [ $5^{\circ}$ ]. Copyright 1999 by the American Physical Society. d) Porous honeycomb networks of anthraquinone molecules on $\mathrm{Cu}(111)$. Image size $26 \mathrm{~nm} \times 15 \mathrm{~nm}$. From [ $\left.9^{\bullet}\right]$. Reprinted with permission from AAAS. 
crystal metal surfaces. This is simply due to the fact that metallic substrates enable the application of a wide variety of different surface-sensitive techniques including STM. Moreover, single crystal metal surfaces represent well-known and well-defined substrates for the deposition of organic molecules. Especially gold surfaces have been used extensively as they are rather inert and easy to clean in ultra-high vacuum environment. Besides gold, especially silver $\left[33^{\bullet}, 37,40^{\bullet}, 41^{\bullet}, 45,46\right]$ and copper $\left[6^{\bullet}, 9^{\bullet}, 47\right]$ have become standard substrates for studying molecular self-assembly.

\section{Cysteine on gold as a model system}

In order to illustrate the above mentioned principle in molecular self-assembly, the adsorption of the natural amino acid cysteine, $\mathrm{HS}-\mathrm{CH}_{2}-\mathrm{CH}\left(\mathrm{NH}_{3}\right)-\mathrm{COOH}$, onto two different faces of gold will be reviewed. It will be demonstrated that all the above mentioned different structural motifs ranging from extended, two-dimensional overlayers, one-dimensional molecular rows, well-defined clusters to porous structures can be achieved with this particular system.

The molecule (see Fig. 5a) is interesting from the point of view that it is the only natural amino acid possessing a mercapto group (HS-) which interacts strongly with gold as known from the archetypical class of alkanethiols studied on $\mathrm{Au}(111)$. Moreover, cysteine possesses an asymmetric carbon atom i.e. a carbon atom that has four different binding partners (indicated by an asterisk in Fig. 5a). This turns cysteine into a chiral molecule with two mirror-symmetric enantiomers, allowing for chiral effects to be studied. The natural and therefore most abundant form is L cysteine. The molecule can be easily evaporated onto the gold substrates by using a Knudsen-type of evaporator as reported previously [31,48-51].

The influence of the molecule-substrate interaction can be illustrated by choosing two different gold substrates, namely $\mathrm{Au}$ (111) and $\mathrm{Au}(110)-(1 \times 2)$ as depicted in Fig. 5b and c, respectively. The hexagonally close-packed $\mathrm{Au}(111)$ surface represents a prime example for an inert substrate. In UHV this surface exhibits the well-known $22 \times \sqrt{ } 3$ reconstruction [52] that is evident from its characteristic herringbone-type appearance. The (110) face of gold reconstructs under UHV conditions into the so-called missing-row reconstruction, where every second close-packed row in the [1-10] direction is missing [53]. In contrast to $\mathrm{Au}(111)$, where the surface atoms are 9-fold coordinated, the $\mathrm{Au}(110)-(1 \times 2)$ surface is highly corrugated, exposing 7-fold coordinated atoms at the surface, which are consequently more reactive than the surface atoms of $\mathrm{Au}(111)$. This difference in surface structure and reactivity is readily reflected in the molecular structure of cysteine evaporated onto these surfaces.

\section{1. $A u(111)$}

In Fig. 6 an STM overview is given on the molecular structures of L cysteine on $\mathrm{Au}(111)$ depending on both substrate temperature and molecular coverage. When cysteine is evaporated onto $\mathrm{Au}(111)$ held at room temperature, unordered
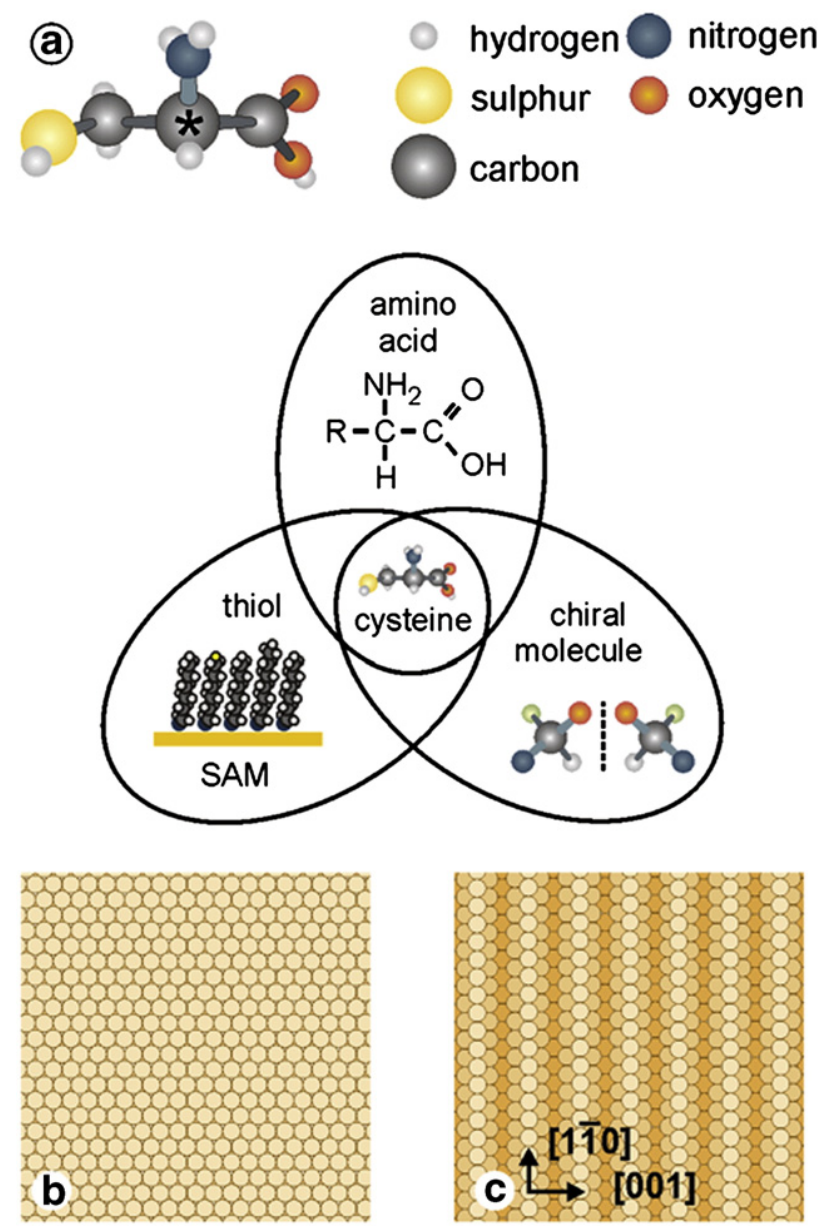

Fig. 5. a) Ball model of the cysteine molecule. b) Model of the hexagonally close-packed $\mathrm{Au}(111)$ surface and c) model of the $(1 \times 2)$ missing-row reconstructed $\mathrm{Au}(110)$ surface.

cysteine islands are observed growing from the step edges and the elbows of the herringbone reconstruction as seen in Fig. 6a. Upon increasing the coverage, these unordered islands grow in size (Fig. 6b), however, no ordering is achieved within the islands. Coexisting with the unordered islands another structure is observed, which is depicted in Fig. 6c. This overlayer does exhibit ordering with a quadratic symmetry. Most strikingly, the herringbone reconstruction is clearly evident underneath this molecular layer, indicating that the herringbone reconstruction remains unperturbed upon molecule evaporation at room temperature. As it is known that chemisorption strongly affects the herringbone reconstruction, this result indicates a noncovalent molecule-substrate interaction. Moreover, this molecular overlayer has been observed with no preferential rotational relationship with respect to the underlying gold substrate, in line with the conclusion of a weak, non-covalent moleculesubstrate interaction.

Higher cysteine coverages evaporated onto Au(111) held at room temperature led to the formation of several different structures, revealing variations of hexagonal patterns as shown in Fig. 6d and e. These structures coexist with the ordered quadratic overlayer, however, they become more pronounced 

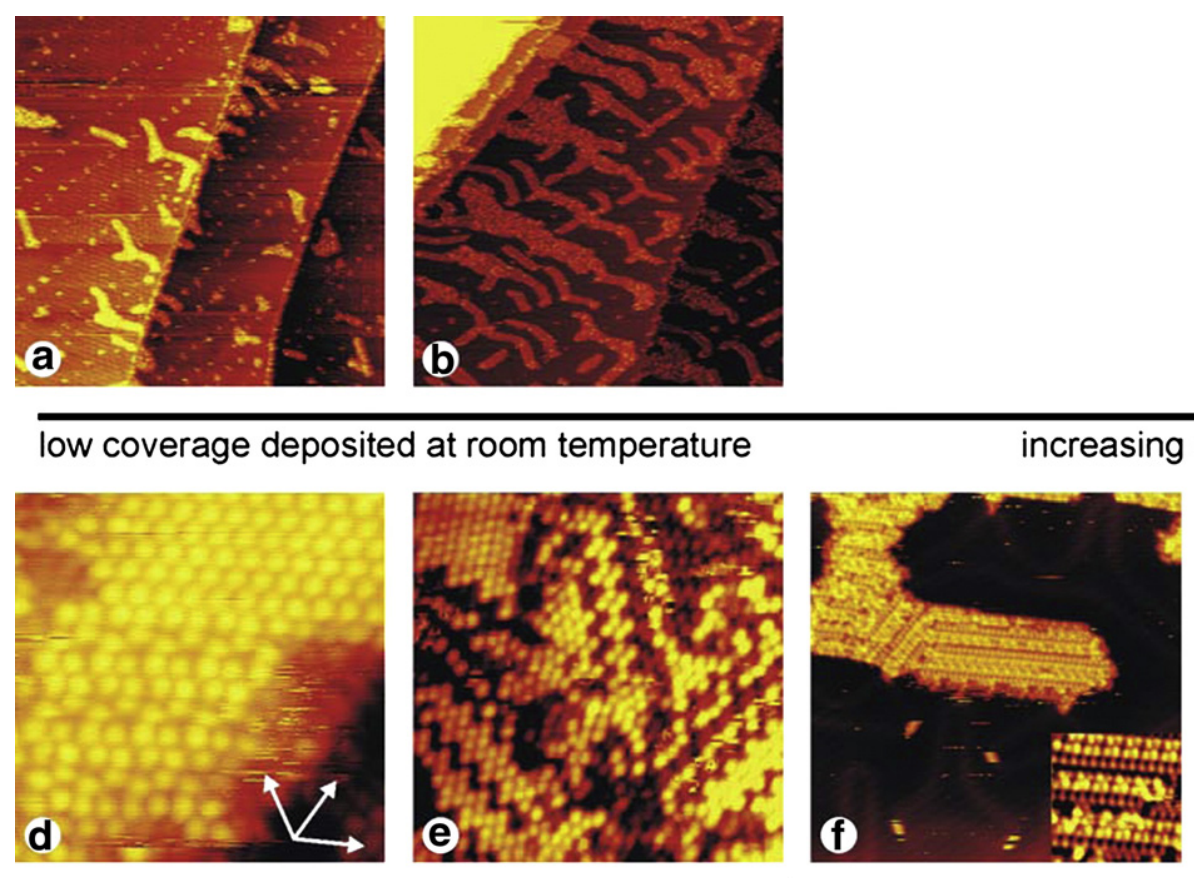

increasing coverage close to $1 \mathrm{ML}$

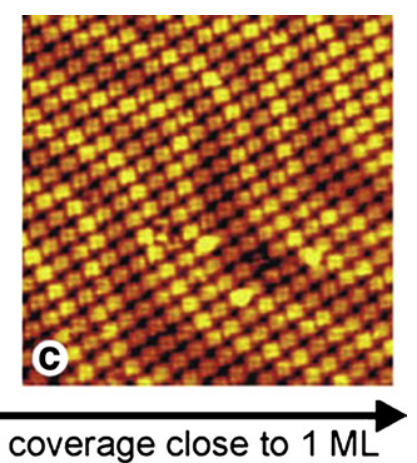

high coverage at room temperature

$\uparrow$

low and high coverage annealed at $380 \mathrm{~K}$

Fig. 6. Overview over the multitude of different molecular structures of L cysteine on $\mathrm{Au}(111)$ in dependence on substrate temperature and surface coverage. a) Upon low-coverage deposition at room temperature, unordered cysteine islands are formed that grow from the step edges and the elbows of the herringbone reconstruction. Image size: $170 \mathrm{~nm} \times 174 \mathrm{~nm}$. b) Upon increased exposure time the islands grow in size. Image size: $111 \mathrm{~nm} \times 116 \mathrm{~nm}$. c) Coexisting with the unordered islands, an ordered overlayer is observed. The underlying herringbone reconstruction is clearly preserved. Image size: $22 \mathrm{~nm} \times 23 \mathrm{~nm}$. d) and e) Molecular structures observed after room-temperature deposition at higher cysteine coverages. Variations of a hexagonal pattern are found. Image sizes $7.6 \mathrm{~nm} \times 8.7 \mathrm{~nm}$ and $15.2 \times 17.4 \mathrm{~nm}$. f) Low cysteine coverage after annealing to $380 \mathrm{~K}$ revealing an internal structure within the cysteine islands. Image size: $38 \mathrm{~nm} \times 44 \mathrm{~nm}$. Insert: $7.6 \mathrm{~nm} \times 8.7 \mathrm{~nm}$. g) High cysteine coverage after annealing to $380 \mathrm{~K}$. Locally the $(\sqrt{ } 3 \times \sqrt{3}) \mathrm{R} 30$ superstructure is formed. Image size $38 \mathrm{~nm} \times 44 \mathrm{~nm}$. Reprinted with permission from Ref. [51]. Copyright 2006 American Chemical Society.

upon increasing the cysteine coverage, suggesting the onset of multilayer formation.

A distinct change in the molecular structure is observed upon annealing the substrate to $380 \mathrm{~K}$. A local order is observed both in the low and high-coverage regime as shown in Fig. $6 \mathrm{f}$ and g, respectively. Now the herringbone reconstruction is indeed perturbed by the presence of the molecular island shown in Fig. 6f, suggesting the transition from physisorbed cysteine to chemisorbed cysteinate molecules involving a covalent sulphur-gold bond between the dehydrogenated mercapto group and the surface $\left(\mathrm{Au}-\mathrm{S}-\mathrm{CH}_{2}-\mathrm{CH}\left(\mathrm{NH}_{3}\right)-\mathrm{COOH}\right)$.

Only upon annealing to $380 \mathrm{~K}$, the high-coverage regime develops the well-known $(\sqrt{ } 3 \times \sqrt{ } 3)$ R3 $30^{\circ}$ overlayer, however, this layer remains imperfect on a large scale (see Fig. 6g). These findings clearly indicate a distinct difference of cysteine adsorbed onto $\mathrm{Au}(111)$ compared to the archetypical system of alkanethiols on this surface.

It is difficult to draw conclusion on the binding properties based on STM data alone. However, the results do indicate that cysteinate formation and covalent bonding on $\mathrm{Au}(111)$ first occurs upon thermal activation. This conclusion has caused some controversy as it is generally believed that alkanethiols readily bind to $\mathrm{Au}(111)$ forming a covalent thiolate-gold bond, which has indeed been observed for cysteine as well, however, deposited onto $\mathrm{Au}(111)$ under liquid environment [54]. However, several recent publications have stressed discrepancies in the current description of alkanethiols on $\mathrm{Au}(111)$, indicating that the picture of thiols on $\mathrm{Au}(111)$ might be more complicated than commonly believed [21,25]. An important aspect is the occurrence of "etch pits" upon alkanethiol deposition even when evaporating under UHV conditions. The puzzle of the etch pits and contradicting experimental and theoretical observations regarding the adsorption site has been addressed by proposing a model involving the removal of gold atoms from the herringbone reconstruction which are subsequently incorporated in an adsorbate-gold complex $[21,25]$. In the present case the situation is clearly different as the herringbone reconstruction can be unambiguously resolved underneath the molecular layer. A possible explanation might be the fact that cysteine is different from prototypical alkanethiols in having both an amino and carboxylic group which gives rise to strong intermolecular interactions which are absent for short-chain alkanethiols.

\section{2. $A u(110)-(1 \times 2)$}

Compared to the situation on $\mathrm{Au}(111)$, the molecular overlayers of cysteine deposited onto $\mathrm{Au}(110)-(1 \times 2)$ reveal 
an even richer variety of geometrical structures. Again, the molecular structures depend on the adsorption state, on the possibility to induce surface restructuring and on the molecular coverage.

When evaporated at $120 \mathrm{~K}$, individual molecules are observed, forming unordered islands consisting of several molecular species as depicted in Fig. 7a. Upon annealing to $270 \mathrm{~K}$ the situation changes remarkably, now revealing identical, monodisperse clusters that are composed of eight subunits as depicted in Fig. 7b [49]. Two centre subunits are surrounded by three units at each side. For clusters formed from L cysteine, the centre units as well as the upper left and lower right corner entities are imaged brighter. This internal structure breaks the mirror symmetry of the system, indicating the chiral nature of the adsorbed molecules. Experiments have revealed that the brighter corner entities are less strongly bound to the cluster compared to the other corner entities [49]. At $270 \mathrm{~K}$ these clusters can be manipulated along the [1-10] direction, leaving behind an unperturbed gold surface, indicating that the clusters are physisorbed to the substrate rather than chemisorbed.

A tentative model for an L cysteine cluster is given in Fig. 7c, showing eight cysteine molecules that form a hydrogen bonded network. All cysteine molecules are physisorbed to the gold surfaces and it is a reasonable starting point to assume that the mercapto group is involved in the physisorption. The detailed adsorption site of the mercapto group, however, cannot be deduced from the STM data shown here. For the model shown in Fig. 7c a bridge position is assumed as adsorption site for the mercapto group. In the centre of the cluster, two cysteine molecules form a dimer through two hydrogen bonds of the carboxylic groups indicated by dotted lines. These cysteine molecules are surrounded by three cysteine molecules on the neighbouring rows. In this model, the amino groups of the two centre molecules form hydrogen bonds with the surrounding cysteine molecules to the lower left and upper right. The three molecules on the neighbouring gold row are interconnected by hydrogen bonds through the carboxylic groups as indicated by the dotted lines. This model readily explains why the upper left and lower right corner entities are imaged brighter and are less strongly bound to the cluster: The corner entities are bound with only one hydrogen bond to the cluster structure and the brighter appearance might be explained by the free oxygen atom that is not involved in hydrogen bond formation.

The situation changes remarkably when annealing to room temperature or depositing the cysteine molecules onto $\mathrm{Au}(110)-(1 \times 2)$ held at room temperature. As depicted in Fig. 7d, a poorly ordered, anisotropic structure is observed. This structure dissolves again upon further annealing to 340-380 K, rearranging into another surface structure that is shown in Fig. 7e. A double-lobe structure is formed with a characteristic $20^{\circ}$ rotation with respect to the close-packed gold rows. The size of each protrusion in the double-lobe feature fits with the size of an individual cysteine molecule, indicating that the double-lobe structure is formed by a cysteine dimer. The rotation of the cysteine dimer depends on the chirality of the adsorbed cysteine molecules. Upon L cysteine adsorption, dimers are formed exhibiting a $20^{\circ}$ clockwise rotation. When evaporating the mirror-imaged enantiomer, D cysteine, the same, but mirror-imaged pairs are formed, revealing a $20^{\circ}$ anticlockwise rotation. Interestingly, when evaporating both enantiomers simultaneously, exclusively homochiral dimers are formed, thus the formation of these cysteine dimers is a molecular-scale example for chiral recognition [48]. Under special tip conditions that occur accidentally, the dimers appear transparent and the underlying gold surface is obtained. As shown in Fig. $7 \mathrm{f}$, holes are observed in the close-packed gold rows, indicating that the molecular dimers formation involves a restructuring of the gold substrate. The expelled gold atoms adsorb to step edges or form added gold rows on to of larger terraces as shown in Fig. $7 \mathrm{~g}$. These added gold rows are only present after dimer formation, indicating that the surface restructuring is driven by the dimer formation. The restructuring readily explains why annealing is necessary for the dimer structure to form as activation energy is needed for the restructuring to occur. Attempts to manipulate the cysteine dimers failed, suggesting a much stronger binding of the dimers to the gold surface compared to the clusters. This is in line with a transition from physisorbed cysteine to chemisorbed cysteinate species. The transition from physisorption to chemisorption might have taken place upon annealing to room temperature, explaining the disappearance of the clusters and the formation of the less ordered structure at room temperature.

When increasing the coverage and annealing to $340 \mathrm{~K}$, another structure is observed to coexist with the molecular dimers. As shown in Fig. 7h, molecular rows are formed running along the [1-10] direction. A zoom into the structure is shown in the insert in Fig. $7 \mathrm{~h}$, revealing a double-row appearance formed from bean-shaped entities of the size of individual cysteine molecules [31]. The STM image suggests that the molecules in the double-row structure interact both along the [001] and [1-10] direction. Density-functional theory (DFT) calculations do, however, indicate that the molecules in the double-row structures only interact along the [001] direction, forming individual cysteine dimers that do not interact along the [1-10] direction (see Fig. 7i). This is an interesting finding as it raises the question of the origin of the one-dimensional growth of the row structure along the [1-10] direction. The solution to this puzzle is again found in a massive substrate restructuring underneath the double-row structure. Both STM and DFT results suggest that two added gold rows underneath the row structure are removed (the original position of these gold rows is indicated by the black arrows in Fig. 7i) [31]. The removal of an atom from a perfect surface is energetically demanding as this involves the reduction of the coordination number of highly coordinated atoms. However, if an atom is removed from an already existing vacancy structure, the energy needed is much smaller. This means that a large energy barrier exists for two cysteine molecules to create a new nucleation site, whereas the growth of an already existing row is energetically more favourable. Thus, the molecule-induced surface rearrangement provides an effective attraction driving the unidirectional growth of the molecular structure. 

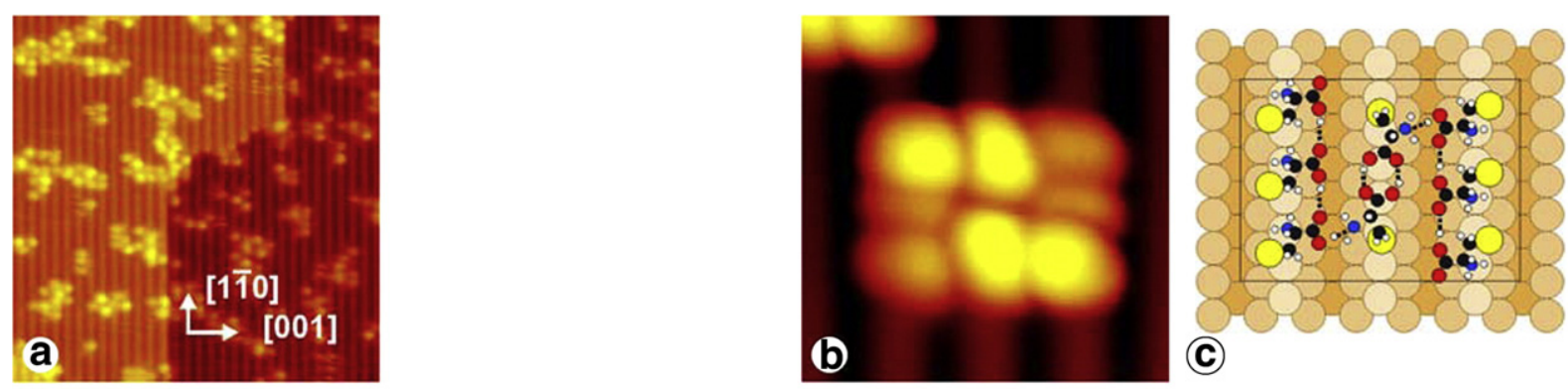

(c)
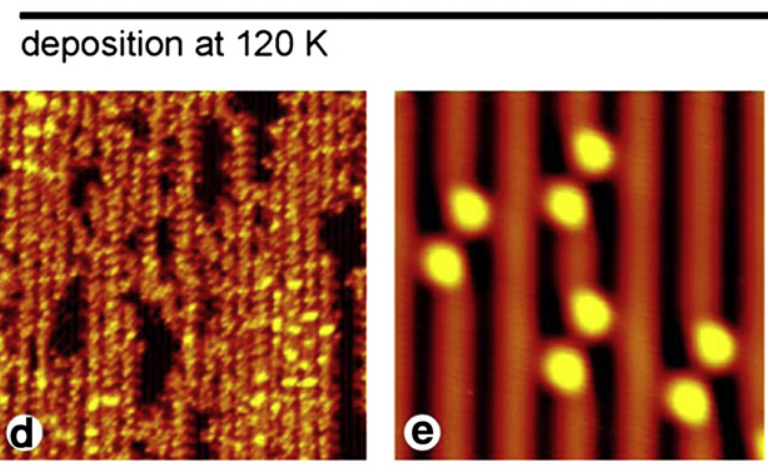

annealing to $270 \mathrm{~K}$
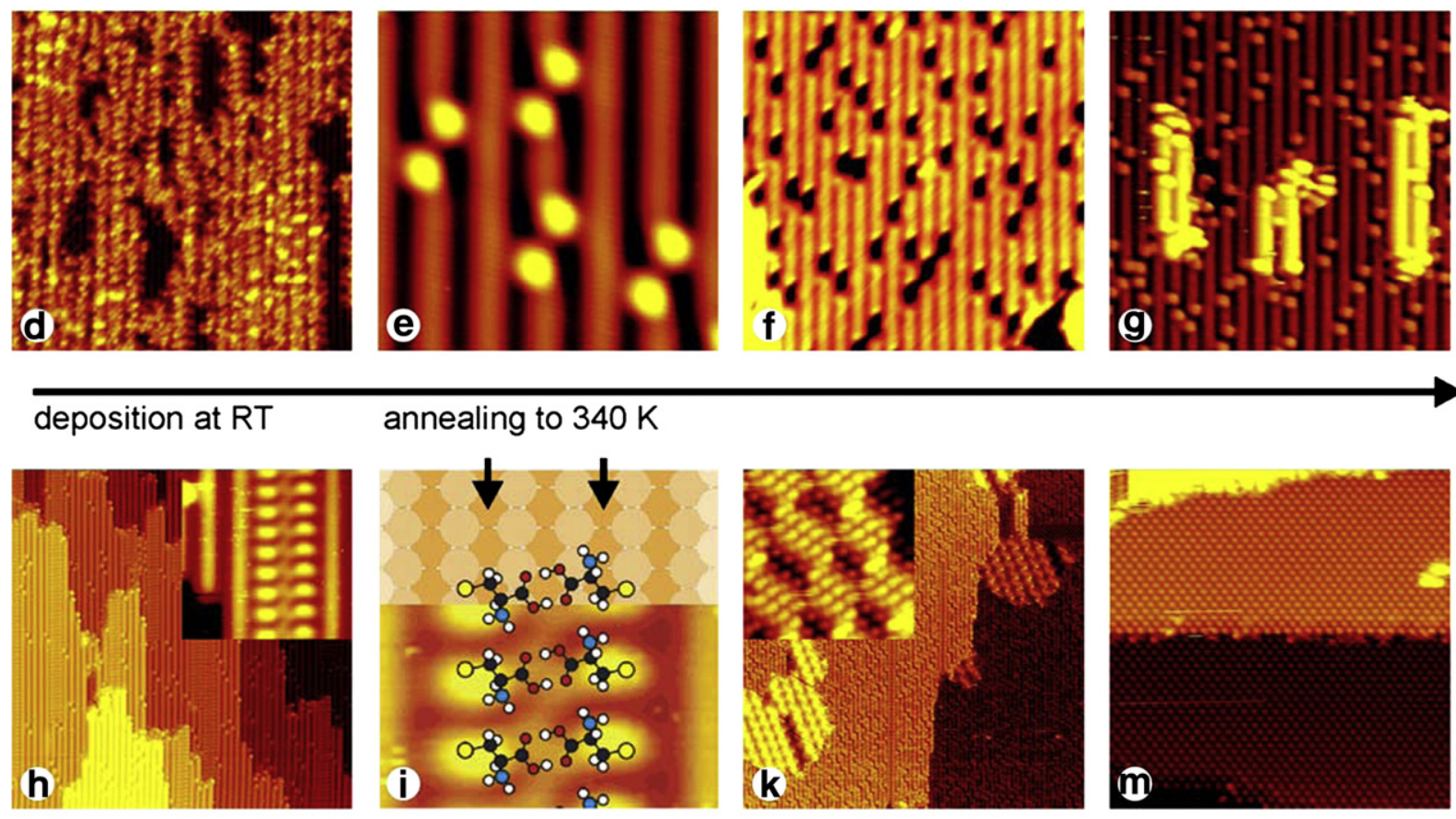

inceasing the coverage
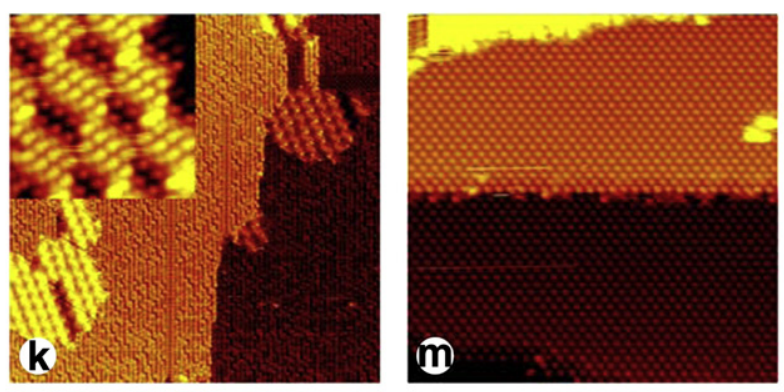

annealing to $440 \mathrm{~K}$

Fig. 7. Overview over the structures observed upon cysteine deposition onto $\mathrm{Au}(110)-(1 \times 2)$. a) Upon deposition onto $\mathrm{Au}(110)-(1 \times 2) \mathrm{kept}$ at $120 \mathrm{~K}$, unordered cysteine agglomerates are observed. Image size $24.5 \mathrm{~nm} \times 24.5 \mathrm{~nm}$. Reprinted with permission from Ref. [49]. Copyright 2003 American Chemical Society. b) When annealing to $270 \mathrm{~K}$ the molecules self-assemble into monodisperse cysteine clusters consisting of eight subunits. For clusters self-assembled from L cysteine, the upper left and lower right corner entities are imaged brighter than the other side entities. Image size $3.2 \mathrm{~nm} \times 3.2 \mathrm{~nm}$. Reprinted with permission from Ref. [49]. Copyright 2003 American Chemical Society. c) Tentative model for an L cysteine cluster as shown in Fig. 7b, d) When annealing to room temperature or depositing cysteine at room temperature, a poorly ordered, anisotropic structure is formed. Image size $40 \mathrm{~nm} \times 40 \mathrm{~nm}$. e) Further annealing to $340 \mathrm{~K}$ leads to the formation of chiral cysteine dimers. Image size $4.9 \mathrm{~nm} \times 5.3 \mathrm{~nm}$. Reprinted by permission from Macmillan Publishers Ltd: Nature Vol. 415 page 891, copyright 2002. f) STM image showing the adsorbate-induced removal of gold atoms: When imaged under special tip conditions, the molecules appear transparent and the underlying gold surface is obtained, revealing holes underneath the dimers. Image size $16.3 \mathrm{~nm} \times 17.7 \mathrm{~nm}$. Reprinted by permission from Macmillan Publishers Ltd: Nature Vol. 415 page 891 , copyright 2002. g) The expelled gold atoms form added gold rows on the terraces. Image size $16.3 \mathrm{~nm} \times 17.7 \mathrm{~nm}$. Reprinted by permission from Macmillan Publishers Ltd: Nature Vol. 415 page 891, copyright 2002. h) Coexisting with the dimers, molecular double rows are formed (D cysteine). Image size $42.5 \mathrm{~nm} \times 42.5 \mathrm{~nm}$. In the insert, a zoom into such a double-row structure is shown. Image size $4 \mathrm{~nm} \times 4 \mathrm{~nm}$. Reprinted figure with permission from [31]. Copyright 2004 by the American Physical Society. i) Most stable cysteine double-row structure as obtained from the DFT calculations superimposed onto a ball model showing the gold surface and on an STM image of the double-row structure. Reprinted figure with permission from [31]. Copyright 2004 by the American Physical Society. k) Upon increasing coverage, a more dense phase grows in size that is coexisting with the dimers and the double-row structure. Here, an island of L cysteine is shown. Image size $62.8 \mathrm{~nm} \times 69.4 \mathrm{~nm}$. In the insert, a zoom into a dense L cysteine structure is shown. Image size $7 \mathrm{~nm} \times 7 \mathrm{~nm}$. Reprinted with permission from Ref. [50]. Copyright 2006 American Chemical Society. m) Eventually, when annealing above $440 \mathrm{~K}$, the molecules desorb dissociatively, leaving behind a c $(4 \times 2)$ sulphur structure. Image size $30 \mathrm{~nm} \times 30 \mathrm{~nm}$.

When the coverage is further increased, yet another structure is observed that coexist with the molecular dimers and molecular double rows [50]. In Fig. 7k an STM image is depicted shown two gold terraces that are covered by both cysteine dimers and double rows. Besides, three islands of a more dense structure are found, a zoom into such an island is shown in the insert. As can be seen in the insert, these islands reveals a rather complex internal structure that seems to involve more than one molecular layer. The islands have been observed to grow predominately from kinked step edges. This has been explained by enantiospecific adsorption of the cysteine molecules to the kink sites, which represent chiral centres at the surface [50]. 


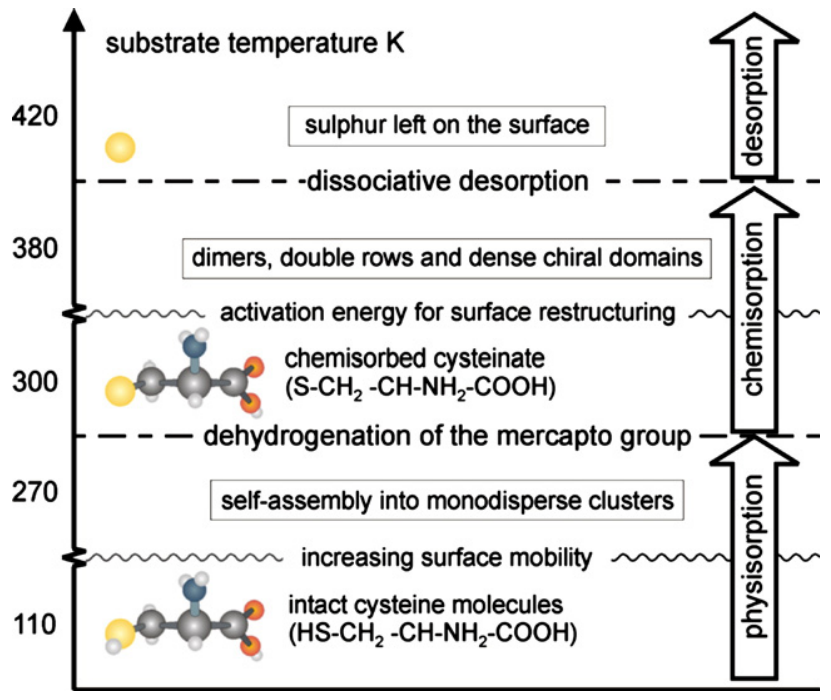

Fig. 8. Summary of the cysteine adsorption structures on $\mathrm{Au}(110)-(1 \times 2)$. Depending on the substrate temperature, the cysteine molecules either physisorb or chemisorb on the surface.

Finally, upon annealing to $440 \mathrm{~K}$, the molecules desorb dissociatively from the surface, leaving atomic sulphur behind on the surface. The sulphur forms a $c(4 \times 2)$ overlayer as depicted in Fig. $7 \mathrm{~m}$. At this stage, the surface is refacetted and exhibits rectangular terraces, again indicating a substantial mass transport upon formation of this $\mathrm{c}(4 \times 2)$ sulphur structure.

A summary of the processes and structures observed upon cysteine adsorption onto $\mathrm{Au}(110)-(1 \times 2)$ is given in Fig. 8. At low temperatures, the molecules physisorb onto the surface and do not have enough mobility to form ordered structures. Only upon annealing to $270 \mathrm{~K}$, the molecules possess enough kinetic energy to diffuse and self-assemble into monodisperse cysteine clusters. Further annealing to room temperature induces the transition from physisorption to chemisorption, which is associated with the disappearance of the clusters. As the chemisorbed species are strongly bound to the gold surface, self-assembly of ordered structures is initially prohibited. First upon annealing to $340-380 \mathrm{~K}$ ordered structures are formed, which involve substrate-induced surface restructuring. Annealing is necessary to supply enough energy to overcome the activation barrier for surface restructuring, which is a prerequisite for the chemisorbed cysteine molecules to selfassemble into ordered structures. Further increasing the annealing temperature leads to the dissociative desorption of the molecules, leaving atomic sulphur behind on the surface.

The relatively simple system of cysteine on $\mathrm{Au}(110)-(1 \times 2)$ thus illustrates the potential of molecular self-assembly for fabricating tailor-made structures, as distinctly different structures can be obtained from the same system simply by changing two parameters namely temperature and molecular coverage.

\section{Summary and outlook}

Molecular self-assembly has developed from an emerging technique to a powerful method for creating well-defined molecular structures. Increasing understanding of the principles behind molecular self-assembly, improving flexibility and control in molecular synthesis as well as extending the range of involved interactions has greatly contributed to this development. Nowadays, an impressive variety of structures can be obtained from predefined components both wellcontrolled and in a predictable fashion.

Future trends in molecular self-assembly include depositing reactive molecular species that react prior to self-assembly. This route is especially interesting for larger molecules that cannot be evaporated by simple heating from a Knudsen-type of evaporator. Such a pre-reaction could also act as a switch to activate the self-assembly process at a well-defined moment. First examples include the activation of molecular species by dehydrogenisation $\left[26^{\circ}\right]$.

Moreover, it is also interesting to design systems that can react after the self-assembly process. In this case, self-assembly can be employed for precise positioning of molecules in order to form a predefined structure. The subsequent reaction can be utilised to produce structures that might be more stable against harsh environmental conditions e.g. changing temperatures as present in real-life applications.

Finally, it is important to extend the studies on molecular self-assembly to non-conductive substrates, as many applications are limited to non-conductive surfaces. Especially for the exciting field of molecular electronics, non-conductive substrates will be mandatory.

\section{Acknowledgements}

This work has been supported by the German Research Foundation (DFG) through the Emmy Noether-programme. Stimulating discussions with Michael Reichling are gratefully acknowledged. I am indebted to Trolle Linderoth and Flemming Besenbacher for their continuous support.

\section{References and recommended reading}

[1] Chaki NK, Vijayamohanan K. Self-assembled monolayers as a tunable platform for biosensor applications. Biosens Bioelectro 2002;17:1-12.

[2] Felhösi I, Kálmán E, Póczik P. Corrosion protection by self-assembly. Russ J Electrochem (Translation of Elektrokhimiya) 2002;38:230-7.

[3] Bain CD, Whitesides GM. Modeling organic-surfaces with self-assembled monolayers. Angew Chem Int Ed Engl 1989;28:506-12.

[4] Laibinis PE, Whitesides GM. Self-assembled monolayers of n-alkanethiolates on copper are barrier films that protect the metal against oxidation by air. J Am Chem Soc 1992;114:9022-8.

[5] Böhringer M, Morgenstern K, Schneider WD, Berndt R, Mauri F, Vita $\mathrm{AD}$, et al. Two-dimensional self-assembly of supramolecular clusters and chains. Phys Rev Lett 1999;83:324-7.

[6] Lukas S, Witte G, Wöll C. Novel mechanism for molecular self-assembly on metal substrates: unidirectional rows of pentacene on $\mathrm{Cu}(110)$ produced by a substrate-mediated repulsion. Phys Rev Lett 2002;88:028301.

[7] Weckesser J, De Vita A, Barth JV, Cai C, Kern K. Mesoscopic correlation of supramolecular chirality in one-dimensional hydrogen-bonded assemblies. Phys Rev Lett 2001;87:096101.

[8] Theobald JA, Oxtoby NS, Phillips MA, Champness NR, Beton PH. Controlling molecular deposition and layer structure with supramolecular surface assemblies. Nature 2003;424:1029-31.

- of special interest.

$\bullet$ of outstanding interest. 
[9] Pawin G, Wong KL, Kwon KY, Bartels L. A homomolecular porous network at a $\mathrm{Cu}(111)$ surface. Science 2006;313:961-2.In this study, substrate-mediated long-range repulsion is employed for network formation.

[10] Stepanow S, Lingenfelder M, Dmitriev A, Spillmann H, Delvigne E, Lin $\mathrm{N}$, et al. Steering molecular organization and host-guest interactions using two-dimensional nanoporous coordination systems. Nat Mater 2004;3:229-33. This is a very interesting paper combining and demonstrating several self-assembly strategies: a nanoporous network is created using complexation of different carboxylic acids with iron atoms. By changing the size and structure of the carboxylic acid, networks with well-defined pore size can be formed. The network is used as a "host" for subsequent C60 "guest" deposition.

[11] Moore GE. Cramming more components onto integrated circuits. Electronics 1965;38:114-6.By that time visionary, by now a historical paper predicting the need for a technology beyond silicon chip manufacturing - the birth of nanotechnology.

[12] Schulz M. The end of the road for silicon? Nature 1999;399:729-30.

[13] Whitesides G, Love C. The art of building small. Sci Am 2001;285:33-41. Descriptive popular science article illustrating conventional lithography and concepts of future nanofabrication.

[14] De Wild M, Berner S, Suzuki H, Ramoino L, Baratoff A, Jung TA. Molecular assembly and self-assembly: molecular nanoscience for future technologies. Ann New York Acad Sci 2003;1006:291-305.

[15] Barth JV, Costantini G, Kern K. Engineering atomic and molecular nanostructures at surfaces. Nature 2005;437:671-9. This review gives a nice overview over tailor-made nanostructures based on atomic and molecular self-assembly, emphasising the difference between selforganisation and self-assembly. Several different strategies for the fabrication of nanostructures are presented including supramolecular engineering employing $\mathrm{H}$-bonding and metal-ligand interactions.

[16] Barth JV. Molecular architectonic on metal surfaces. Annu Rev Phys Chem 2007;58:375-407. This paper nicely summarizes central self-assembly principles. Recent advances in molecular self-assembly on metal surfaces are reviewed, involving different types of molecular interactions. Finally, examples of hierarchical self-assembly are presented.

[17] De Feyter S, De Schryver FC. Self-assembly at the liquid/solid interface: STM reveals. J Phys Chem B 2005;109:4290-302. Review illustrating the richness of two-dimensional structures of organic molecules at the liquidsolid interface. Also, the important role of the solvent in molecular selfassembly at the liquid-solid interface is stressed.

[18] Lackinger M, Griessl S, Kampschulte L, Jamitzky F, Heckl WM. Dynamics of grain boundaries in two-dimensional hydrogen-bonded molecular networks. Small 2005;1:532-9.

[19] Whitesides GM, Mathias JP, Seto CT. Molecular self-assembly and nanochemistry: a chemical strategy for the synthesis of nanostructures. Science 1991;254:1312-9. This by now historical paper "set the stage" for basically all later work on molecular self-assembly discussing chemical strategies for synthesising nanostructures.

[20] Röder H, Hahn E, Brune H, Bucher JP, Kern K. Building one- and twodimensional nanostructures by diffusion-controlled aggregation at surfaces. Nature 1993;366:141-3.

[21] Maksymovych P, Sorescu DC, Yates J, John T. Gold-adatom-mediated bonding in self-assembled short-chain alkanethiolate species on the $\mathrm{Au}$ (111) surface. Phys Rev Lett 2006;97:146103-4.

[22] Schunack M, Petersen L, Kühnle A, Lægsgaard E, Stensgaard I, Johannsen I, et al. Anchoring of organic molecules to a metal surface: $\mathrm{HtBDC}$ on $\mathrm{Cu}$ (110). Phys Rev Lett 2001;86:456-9.

[23] Chen Q, Richardson NV. Surface facetting induced by adsorbates. Prog Surf Sci 2003;73:59-77.

[24] Schreiber F. Structure and growth of self-assembling monolayers. Prog Surf Sci 2000;65:151-256. Comprehensive review presenting structural phases and growth of thiols on $\mathrm{Au}(111)$.

[25] Mazzarello R, Cossaro A, Verdini A, Rousseau R, Casalis L, Danisman $\mathrm{MF}$, et al. Structure of a $\mathrm{CH}_{3} \mathrm{~S}$ monolayer on $\mathrm{Au}(111)$ solved by the interplay between molecular dynamics calculations and diffraction measurements. Phys Rev Lett 2007;98:016102.In this combined experimental and theoretical investigation the long-standing puzzle of the methylthiolate adsorption site has been revisited. Both experiments and theory are in agreement with a model involving a reconstruction of the gold surface resulting in gold adatoms

[26] Stöhr M, Wahl M, Galka CH, Riehm T, Jung TA, Gade LH. Controlling molecular assembly in two dimensions: the concentration dependence of thermally induced 2D aggregation of molecules on a metal surface. Angew Chem 2005;117:7560-4. In this publication, the molecules under investigation are "activated" by annealing to $570 \mathrm{~K}$. Only after this activation, the molecular species are able to form hydrogen bonding. Moreover, the observed self-assembled structures are coverage-dependent: upon increasing the coverage from 0.1 to $1 \mathrm{ML}$, three different molecular structures are formed.

[27] Otero R, Schöck M, Molina LM, Lægsgaard E, Stensgaard I, Hammer B, et al. Guanine quartet networks stabilized by cooperative hydrogen bonds. Angew Chem Int Ed 2005;44:2270-5.

[28] Yokoyama T, Yokoyama S, Kamikado T, Okuno Y, Mashiko S. Selective assembly on a surface of supramolecular aggregates with controlled size and shape. Nature 2001;413:619-21. One of the very first papers illustrating the potential of dipole-dipole interactions on molecular selfassembly. By controlling the number and position of substituents on a porphyrin molecule, molecular structures are formed in a predicable fashion.

[29] Messina P, Dmitriev A, Lin N, Spillmann H, Abel M, Barth JV, et al. Direct observation of chiral metal-organic complexes assembled on a $\mathrm{Cu}(100)$ surface. J Am Chem Soc 2002;124:14000-1.

[30] Repp J, Moresco F, Meyer G, Rieder KH, Hyldgaard P, Persson M. Substrate mediated long-range oscillatory interaction between adatoms: $\mathrm{Cu} / \mathrm{Cu}(111)$. Phys Rev Lett 2000;85:2981-4.

[31] Kühnle A, Molina LM, Linderoth TR, Hammer B, Besenbacher F. Growth of unidirectional molecular rows of cysteine on $\mathrm{Au}(110)-(1 \times 2)$ driven by adsorbate-induced surface rearrangements. Phys Rev Lett 2004;93:086101.

[32] Taylor JB, Beton PH. Kinetic instabilities in the growth of one dimensional molecular networks. Phys Rev Lett 2006;97:236102.

[33] De Wild M, Berner S, Suzuki H, Yanagi H, Schlettwein D, Ivan S, et al. A novel route to molecular self-assembly. Self-intermixed monolayer phases. Chem Phys Chem 2002;3:881-5. One of the very first studies on selfassembly of coadsorbed molecular species. The influence of relative coverage is presented, nicely illustrating the transition from a close-packed C60 monolayer through transition states to an open Sub-Pc honeycomb network.

[34] Zhdanov VP. Ostwald ripening of close-packed and honeycomb islands during coadsorption. Phys Rev B 2007;76:033406.

[35] Glöckler K, Seidel C, Soukopp A, Sokolowski M, Umbach E, Böhringer $\mathrm{M}$, et al. Highly ordered structures and submolecular scanning tunnelling microscopy contrast of PTCDA and DM-PBDCI monolayers on $\mathrm{Ag}(111)$ and $\mathrm{Ag}(110)$. Surf Sci 1998;405:1-20.

[36] Lorenzo MO, Baddeley CJ, Muryn C, Raval R. Extended surface chirality from supramolecular assemblies of adsorbed chiral molecules. Nature 2000;404:376-9.In this paper, the formation of extended chiral domains is reported.

[37] Berner S, De Wild M, Ramoino L, Ivan S, Baratoff A, Güntherodt HJ, et al. Adsorption and two-dimensional phases of a large polar molecule: subphthalocyanine on $\operatorname{Ag}(111)$. Phys Rev B 2003;68:115410.

[38] Kossev I, Reckien W, Kirchner B, Felder T, Nieger M, Schalley CA, et al. Highly ordered 2D hydrogen-bonded structures of a tetralactam macrocycle on the Au(111) surface. Adv Funct Mater 2007;17:513-9.

[39] Oison V, Koudia M, Abel M, Porte L. Influence of stress on hydrogenbond formation in a halogenated phthalocyanine network. Phys Rev B 2007;75:035428

[40] Eremtchenko M, Schaefer JA, Tautz FS. Understanding and tuning the epitaxy of large aromatic adsorbates by molecular design. Nature 2003;425:602-5

[41] Barth JV, Weckesser J, Cai C, Günter P, Bürgi L, Jeandupeux O, et al. Building supramolecular nanostructures at surfaces by hydrogen bonding. Angew Chem Int Ed 2000;39:1230-4.

[42] Lopinski G, Wayner D, Wolkow R. Self-directed growth of molecular nanostructures on silicon. Nature 2000;406:48-51. 
[43] Okawa Y, Aono M. Materials science: nanoscale control of chain polymerization. Nature 2001;409:683-4.

[44] Dmitriev A, Spillmann H, Lin N, Barth JV, Kern K. Modular assembly of two-dimensional metal-organic coordination networks at a metal surface. Angew Chem Int Ed 2003;42:2670-3.

[45] Tautz FS, Sloboshanin S, Shklover V, Scholz R, Sokolowski M, Schaefer JA, et al. Substrate influence on the ordering of organic submonolayers: a comparative study of PTCDA on $\operatorname{Ag}(110)$ and $\operatorname{Ag}(111)$ using HREELS. Appl Surf Sci 2000;166:363-9.

[46] Bonifazi D, Spillmann H, Kiebele A, de Wild M, Seiler P, Cheng F, et al. Self-assembly on surfaces: supramolecular patterned surfaces driven by cooperative assembly of $\mathrm{C} 60$ and porphyrins on metal substrates. Angew Chem Int Ed 2004;43:4759-63.

[47] Chen Q, Frankel DJ, Richardson NV. Self-assembly of adenine on $\mathrm{Cu}$ (110) surfaces. Langmuir 2002;18:3219-25.

[48] Kühnle A, Linderoth TR, Hammer B, Besenbacher F. Chiral recognition in dimerization of adsorbed cysteine observed by scanning tunneling microscopy. Nature 2002;415:891-3.

[49] Kühnle A, Linderoth TR, Besenbacher F. Self-assembly of monodispersed, chiral nanoclusters of cysteine on the $\mathrm{Au}(110)-(1 \times 2)$ surface. J Am Chem Soc 2003;125:14680-1.
[50] Kühnle A, Linderoth TR, Besenbacher F. Enantiospecific adsorption of cysteine at chiral kink sites on $\mathrm{Au}(110)-(1 \times 2)$. J Am Chem Soc 2006;128:1076-7.

[51] Kühnle A, Linderoth TR, Schunack M, Besenbacher F. L-cysteine adsorption structures on $\mathrm{Au}(111)$ investigated by scanning tunneling microscopy under ultrahigh vacuum conditions. Langmuir 2006;22:2156-60.

[52] Barth JV, Brune H, Ertl G, Behm RJ. Scanning tunneling microscopy observations on the reconstructed $\mathrm{Au}(111)$ surface: atomic structure, longrange superstructure, rotational domains, and surface defects. Phys Rev B 1990;42:9307-18.

[53] Gritsch T, Coulman D, Behm RJ, Ertl G. A scanning tunneling microscopy investigation of the structure of the $\mathrm{Pt}(110)$ and $\mathrm{Au}(110)$ surfaces. Surf Sci 1991;257:297-306.

[54] Dakkouri AS, Kolb DM, Edelstein-Shima R, Mandler D. Scanning tunneling microscopy study of L-cysteine on $\mathrm{Au}(111)$. Langmuir 1996;12:2849-52. 